\title{
Optimized Homonuclear Carr-Purcell-Type Dipolar Mixing Sequences
}

\author{
Frank Kramer, ${ }^{*}$ Wolfgang Peti, $†$ Christian Griesinger,$\dagger \dagger$ and Steffen J. Glaser*,1 \\ * Institut für Organische Chemie und Biochemie II, Technische Universität München, Lichtenbergstrasse 4, D-85747 Garching, Germany; $\dagger$ Institut für \\ Organische Chemie, J. W. Goethe-Universität Frankfurt, Marie-Curie-Strasse 11, D-60439 Frankfurt, Germany; and $\ddagger$ Max-Plank Institute \\ for Biophysical Chemistry, Am Faßberg 11, D-37077 Göttingen, Germany
}

Received September 5, 2000

\begin{abstract}
Modified phase-cycled Carr-Purcell-type multiple-pulse sequences are optimized for coherence and polarization transfer in homonuclear Hartmann-Hahn experiments applied to partially oriented samples. Compared to previously suggested experiments, the sequences yield considerably improved scaling factors for residual dipolar coupling constants, resulting in improved transfer efficiency. $\odot 2001$ A cademic Press
\end{abstract}

Key Words: Hartmann-Hahn transfer; residual dipolar couplings; C arr-Purcell sequences; dipolar mixing; T OC SY, D C OSY.

\section{INTRODUCTION}

Residual dipolar couplings of partially oriented molecules yield valuable structural information and can be used to correlate distant nuclear spins in high-resolution NMR (1-6). Homonuclear Hartmann-Hahn (TOCSY) transfer (7-9) through residual dipolar couplings has found useful applications in biological NMR $(5,6)$. The corresponding experiments based on $J$ couplings (isotropic mixing) (7) and on dipolar couplings (dipolar mixing) have several common features, but also distinct differences $(5,9,10)$. In order to create energy-matched conditions, both isotropic and dipolar mixing experiments rely on the effective suppression of offset terms. However, the specific forms of the isotropic and dipolar coupling tensors give rise to very different transfer dynamics $(5,9,11,12)$. In addition, the different transformation properties under rotations can result in very different scaling properties of isotropic and dipolar coupling constants under the same multiple-pulse sequence $(10,13)$. As the rate of polarization or coherence transfer is directly proportional to the size of the (scaled) effective coupling constants, large scaling factors are highly desirable in order to minimize relaxation effects. Here, we present optimized dipolar mixing sequences with useful bandwidths and considerably improved scaling factors compared to previously suggested sequences.

\footnotetext{
${ }^{1}$ To whom correspondence should be addressed. E-mail: glaser@ ch.tum.de.
}

\section{THEORY}

We consider a system of dipolar-coupled spins with coupling terms of the form

$$
H_{d, k l}=2 \pi D_{k l}\left(I_{k x} I_{l x}+I_{k y} I_{l y}-2 I_{k z} I_{l z}\right)
$$

for each spin pair $(k l)$. The residual dipolar coupling constant is given by (14)

$$
D_{k l}=S \frac{\mu_{0} \gamma_{k} \gamma_{l} \hbar}{8 \pi^{2} r_{k l}^{3}}\left[A_{a}\left(3 \cos ^{2} \theta-1\right)+\frac{3}{2} A_{r}\left(\sin ^{2} \theta \cos 2 \phi\right)\right],
$$

where $S$ is a generalized order parameter, $\gamma_{k}$ is the gyromagnetic ratio of spin $k, r_{k l}$ is the internuclear distance, and $A_{a}$ and $A_{r}$ are the axial and rhombic components of the molecular alignment tensor, in whose principle axis system the bond vector is defined by the cylindrical coordinates $\theta$ and $\phi$. If the form of the coupling tensor is conserved by the multiple-pulse sequence (10), the effective coupling terms can be expressed as

$$
H_{d, k l}^{e f f}=2 \pi D_{k l}^{e f f}\left(I_{k x^{\prime}} I_{l x^{\prime}}+I_{k y^{\prime}} I_{l y^{\prime}}-2 I_{k z^{\prime}} I_{l z^{\prime}}\right)
$$

The effective coupling constant $D_{k l}^{e f f}$ is related to the residual dipolar coupling constant $D_{k l}$ by a scaling factor $s_{k l}$ that is defined as

$$
s_{k l}=\frac{D_{k l}^{e f f}}{D_{k l}}
$$

with $\left|s_{k l}\right| \leq 1$. Because in the principal axis system of the effective Hamiltonian $\mathrm{H} \quad \begin{gathered}\text { eff } \\ d, k l\end{gathered}$ the transfer of transverse $\left(x^{\prime}, y^{\prime}\right)$ magnetization is markedly different from the transfer of longitudinal $\left(z^{\prime}\right)$ magnetization, we will frequently translate transfer functions $T_{\alpha}$ (for $\alpha=x, y$, or $z$ ) from the rotating frame to transfer functions $T_{\alpha}$ (for $\alpha=x^{\prime}, y^{\prime}$, or $z^{\prime}$ ) in the principal axis frame, in order to facilitate comparison. Compared to the MLEV16 sequence (15) with an on-resonance dipolar scaling factor of $\left|s_{k l}\right|=1 / 4$ (13), the phase-alternating sequences WALTZ16 (16) and DIPSI-2 (17) yield a more favorable on-resonance 


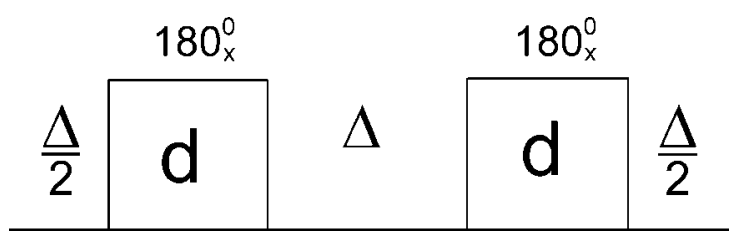

FIG. 1. Carr-Purcell-type basis sequence of duration $\tau_{c y c}=2(d+\Delta)$, consisting of two $180^{\circ}$ pulses (of duration $d$ ) surrounded by delays (of duration $\Delta / 2)$.

scaling factor of $\left|s_{k l}\right|=1 / 2$ (13). In principle, a scaling factor $\left|s_{k l}\right|=1$ can be achieved by Carr-Purcell-type sequences (18), consisting of a series of ideal, hard $180^{\circ}$ pulses (13). However, previously such sequences have not been considered for applications in practical dipolar mixing sequences, because infinitely short pulses cannot be implemented in practice. In fact, pulse sequences of this type were suggested in the seminal paper on homonuclear isotropic mixing experiments based on $J$ couplings (7), but never found widespread use because of their limited bandwidth (9). However, as will be shown below, series of properly phase-cycled $180^{\circ}$ pulses with finite amplitudes and optimized durations of the delays between the pulses form an attractive class of homonuclear dipolar mixing sequences. We refer to this class of mixing sequences as modified phase-cycled Carr-Purcell-type (MOCCA) sequences.

The basis cycle of a Carr-Purcell sequence (characterized by the length of the $180^{\circ}$ pulse $d$ and the duration of the windows $\Delta$ ) is shown in Fig. 1. In a toggling frame defined by the rf sequence and considering just zero-order effects, offset terms average to zero, creating energy-matched conditions (9). The zero-order average dipolar coupling terms can be derived in a straightforward way (see Appendix) and have the form

$$
\begin{aligned}
H^{-}{ }_{d, k l} & =2 \pi\left\{D_{k l}^{e f f, x} I_{k x} I_{l x}+D_{k l}^{e f f, y} I_{k y} I_{l y}+D_{k l}^{e f f, z} I_{k z} I_{l z}\right\} \\
& \equiv 2 \pi D_{k l}\left\{I_{k x} I_{l x}+\frac{2 \Delta-d}{2(d+\Delta)} I_{k y} I_{l y}-\frac{4 \Delta+d}{2(d+\Delta)} I_{k z} I_{l z}\right\}
\end{aligned}
$$

This (zero-order) average Hamiltonian approximates a valid effective Hamiltonian $\mathrm{H}$ eff provided that the cycle time $\tau_{c y c}$ of the sequence fulfills

$$
\tau_{c y c}=2(d+\Delta) \ll \frac{1}{v_{o f f}^{\max }},
$$

where $v_{\text {off }}^{\max }$ is the largest offset in the spin system $(19,20)$. For $\Delta \ll d$, the average coupling term $\mathrm{H}^{-} d, k l$ in Eq. [5] has the form of $\mathrm{H}_{d, k l}^{e f f}$ in Eq. [3] with $D_{k l}^{\text {eff }}=-D_{k l} / 2$ and $x^{\prime}=z, y^{\prime}=y$ and $z^{\prime}=x$. This limit corresponds to the case of CW irradiation $(\Delta=0)$ with $s_{k l}=-1 / 2$ and has the same on-resonance average Hamiltonian as DIPSI-2 and WALTZ-16 (13). On the other hand, $\mathrm{H}^{-}{ }_{d, k l}=\mathrm{H} \quad d, k l$ with the most favorable scaling factor $s_{k l}=1$ and $x^{\prime}=x, y^{\prime}=y$, and $z^{\prime}=z$ are found in the limit of ideal pulses $(\Delta \gg d)$. For an effective Hamiltonian of the form of Eq. [5], the transfer functions of $x, y$, and $z$ magnetization are given by (9)

$$
T_{\alpha}=\sin \left(\pi D_{k l}^{e f f, \beta} \tau\right) \sin \left(\pi D_{k l}^{e f f, \gamma} \tau\right),
$$

where $\{\alpha, \beta, \gamma\}$ are permutations of $\{x, y, z\}$.

For the two limiting cases $\Delta \ll d$ and $\Delta \gg d$, where the effective Hamiltonian has the form of Eq. [3] the transfer functions reduce to the following form in the principle axis system $\left\{x^{\prime}, y^{\prime}\right.$, $\left.z^{\prime}\right\}$ of the effective dipolar coupling tensor:

$$
\begin{aligned}
& T_{x^{\prime}}=T_{y^{\prime}}=-\sin \left(\pi D_{k l}^{e f f} \tau\right) \sin \left(2 \pi D_{k l}^{e f f} \tau\right), \\
& T_{z^{\prime}}=\sin ^{2}\left(\pi D_{k l}^{e f f} \tau\right) .
\end{aligned}
$$

For $\Delta \ll d, D_{k l}^{e f f, x}=D_{k l}$ and $D_{k l}^{e f f, y}=D_{k l}^{e f f, z}=-D_{k l} / 2$ in Eqs. [5] and [7] (corresponding to $D_{k l}^{\text {eff }}=-D_{k l} / 2$ in Eqs. [3] and [8]). In this case the transfer curves $T_{x^{\prime}}=T_{y^{\prime}}=T_{y}=T_{z}$ (see Fig. 2B, thin curve) reach extrema at approximately $\tau=$ $0.3 /\left|D_{k l}^{\text {eff }}\right| \approx 0.6 /\left|D_{k l}\right|$ and $T_{z^{\prime}}=T_{x}$ (see Fig. 2A, thin curve) has a maximum at $\tau=1 /\left(2\left|D_{k l}^{e f f}\right|\right) \approx 1 /\left|D_{k l}\right|$. These transfer curves are identical to the on-resonance transfer functions found for DIPSI-2. In contrast, transfer times are reduced by a factor of 2 for $\Delta \gg d$, where $D_{k l}^{e f f, x}=D_{k l}^{e f f, y}=D_{k l}$ and $D_{k l}^{e f f, z}=-2 D_{k l}$ in Eqs. [5] and [7] (corresponding to $D_{k l}^{e f f}=D_{k l}$ in Eqs. [3] and [8]). In this case, the transfer curves $T_{x^{\prime}}=T_{y^{\prime}}=T_{x}=T_{y}$ (see Fig. 2B, dashed thin curve) reach extrema approximately at $\tau=0.3 /\left|D_{\text {eff }}\right| \approx 0.3 /|D|$ and $T_{z^{\prime}}=T_{z}$ (see Fig. 2A, dashed thin curve) has a maximum at $\tau=1 /\left(2\left|D_{\text {eff }}\right|\right) \approx 1 /(2|D|)$. This is the most desirable limit with the fastest transfer rate.

In order to increase the active offset range of dipolar transfer, we now consider modified phase-cycled Carr-Purcelltype sequences, consisting of an inversion element $R_{\varphi}=(\Delta / 2-$ $\left.180_{\varphi}^{\circ}-\Delta / 2\right)$ that is subject to commonly used supercyles. In order to use a concise notation, we refer to the various members of the MOCCA family by indicating the cycle (or supercycle) as an extension. For example, the MOCCA-M16 and the MOCCA-XY16 sequences consist of 16 elements $R_{\varphi}$ with phases $0,0, \pi, \pi, \pi, 0,0, \pi, \pi, \pi, 0,0,0, \pi, \pi, 0$ corresponding to the MLEV-16 supercycle (15) and $0, \pi / 2,0, \pi / 2$, $\pi / 2,0, \pi / 2,0, \pi, 3 \pi / 2, \pi, 3 \pi / 2,3 \pi / 2, \pi, 3 \pi / 2, \pi$ corresponding to the XY-16 supercycle $(21,22)$, respectively. Similarly, in the MOCCA-M4P9 sequence the basic inversion element $R_{\varphi}$ is expanded according to the M4P9 supercycle $(23,24)$. Note that the average coupling term given in Eq. [5] is not valid for MOCCA-XY16 and MOCCA-M4P9 because also phases $\varphi$ other than 0 or $\pi$ are used in these sequences. However, Eq. [5] is still valid for MOCCA-M16 if rf inhomogeneity is neglected and the on-resonance case is considered. For MOCCA-XY16 

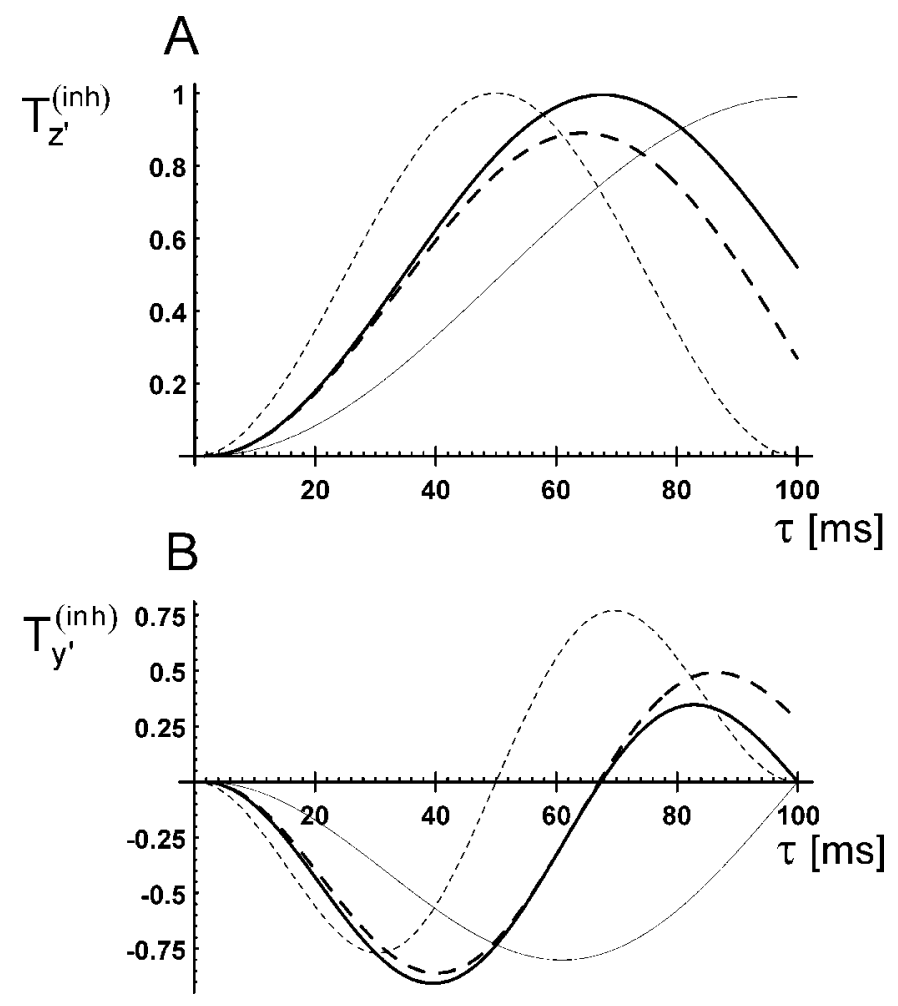

FIG . 2. Time dependence of characteristic ideal transfer functions $T_{\alpha}(\tau)$ and of simulated transfer functions $T_{\alpha}^{i n h}(\tau)$ for the best MOCCA sequences (bold type in Table 1) in the presence of rf inhomogeneity. (A) Theoretical polarization transfer function $T_{z^{\prime}}(\tau)$ (Eq. [8]) based on the average Hamiltonian $\mathrm{H}^{-}{ }_{d, k l}$ (Eq. [5]) for $\Delta \ll d\left(z^{\prime}=x\right.$, thin curve) and $\Delta \gg d\left(z^{\prime}=z\right.$, dashed thin curve) compared to the realistic transfer function $T_{z}^{i n h}(\tau)$, in the presence of $\mathrm{rf}$ inhomogeneity for MOCCA-XY16 (bold curve) with $\Delta=2.2 d$ and MOCCAM4P9 (dashed bold curve) with $\Delta=2.4 d$ (see Table 1). (B) Theoretical transfer function $T_{x^{\prime}}(\tau)$ (Eq. [8]) based on the average Hamiltonian $\mathrm{H}^{-}{ }_{d, k l}$ (Eq. [5]) for $\Delta \ll d\left(x^{\prime}=z\right.$, thin curve $)$ and $\Delta \gg d\left(x^{\prime}=x\right.$, dashed thin curve $)$ compared to the realistic transfer function $T_{y}^{i n h}(\tau)$, in the presence of rf inhomogeneity for MOCCA-M16 (bold curve) with $\Delta=2.2 d$ and MOCCA-M4P9 (dashed bold curve) with $\Delta=2.4 d$ (see Table 1). The on-resonance transfer functions $T_{x}=T_{z^{\prime}}$ and $T_{y}=T_{z}=T_{x^{\prime}}$ of DIPSI-2 are identical to the solid thin curves shown in $\mathrm{A}$ and $\mathrm{B}$.

the average coupling term on resonance is given by

$$
H^{-}{ }_{d, k l}=2 \pi D_{k l}\left(\frac{d / 4+\Delta}{d+\Delta}\right)\left\{I_{k x} I_{l x}+I_{k y} I_{l y}-2 I_{k z} I_{l z}\right\},
$$

since the phase cycle leads to an average between Eq. [5] and Eq. [5] with $x$ and $y$ interchanged.

In contrast to Eq. [5], this average coupling term has the form of Eq. [3] for all values of $\Delta$ with

$$
s_{k l}=\frac{d / 4+\Delta}{d+\Delta}
$$

and $x^{\prime}=x, y^{\prime}=y$, and $z^{\prime}=z$. For all MOCCA-type sequences, $\Delta \gg d$ is desirable in order to maximize the on-resonance scaling factor. However, the maximum value of $\Delta$ is limited by Eq. [6]. Hence, based on the simple zero-order average
Hamiltonian arguments given above and considering that the $180^{\circ}$ pulse duration $d=1 / 2 v_{r f}$ is limited by the maximum available rf amplitude $v_{r f}^{\max }$, the optimal duration of the delay $\Delta$ is expected to be found in the range

$$
\frac{1}{v_{r f}^{\max }}<\Delta<\frac{1}{v_{o f f}^{\max }} .
$$

For example, Fig. 3 shows numerical simulations of the offset dependence of the transfer efficiency of the MOCCA-M16 sequence with an $\mathrm{rf}$ amplitude of $12.5 \mathrm{kHz}$ for a range of delays $\Delta$ and $D=D_{k l}=10 \mathrm{~Hz}$. Realistic transfer functions $T_{\alpha}^{i n h}(\tau)$ in the presence of $\mathrm{rf}$ inhomogeneity were simulated using an extended version of the program SIMONE (25) by superimposing a series of ideal transfer functions $T_{\alpha}(\tau)$ for different nominal rf amplitudes, weighted by a Gaussian rf inhomogeneity distribution with a width of $10 \%$ at half-height (9). For the MOCCA-M16 sequence the on-resonance effective Hamiltonian is identical to Eq. [5]. Based on this effective Hamiltonian and the theoretical transfer function given in Eq. [7], the transfer amplitude $T_{x}(0.3 / D)$ is given by 0.205 , $0.128,0,-0.448,-0.613$, and -0.698 for a delay $\Delta$ of $1,10,20$, 80,160 , and $320 \mu s$, respectively. These values are indeed found for the on-resonance case $\left(v_{1}=v_{2}=0\right)$ in Fig. 3. As expected from Eq. [5] the on-resonance transfer $T_{x}^{i n h}(0.3 / D)$ is positive (Eq. [8]) for $\Delta \ll d$ (see Figs. 3A and 3B) and negative (Eq. [7]) for $\Delta \gg d$ (see Figs. 3E and 3F). In Fig. 3C no transfer is observed, because the average Hamiltonian for $\Delta=d / 2$ is given by $\mathrm{H}^{-}{ }_{d, k l}=2 \pi D_{k l}\left\{I_{k x} I_{l x}-I_{k z} I_{l z}\right\}$ (see Eq. [5]), which according to Eq. [7] does not give rise to transfer of $x$ magnetization. Note that for $\Delta \gg d$ efficient coherence transfer is limited to a relatively narrow region along the diagonal, with $\left|v_{1}-v_{2}\right| \ll 1 / \Delta$; e.g., in Fig. 3F, the transfer is limited to $\left|v_{1}-v_{2}\right|<1.5 \mathrm{kHz}$. This example demonstrates the need to optimize the delay $\Delta$ for various members of the MOCCA family in order to find an optimal compromise between usable bandwidth and large scaling factors $s_{k l}$.

\section{OPTIMIZE D SEQUENCES}

The delay $\Delta$ was optimized for various MOCCA sequences to maximize the dipolar transfer efficiency in a defined offset region using an extended version of the program SIMONE $(9,25)$. The results for a target spectral width of $8 \mathrm{kHz}$ and different magnetization components are summarized in Table 1 for MOCCA-XY16, MOCCA-M16, and MOCCA-M4P9 for an rf amplitude $v_{r f}=12.5 \mathrm{kHz}$. In addition to the optimized pulse sequence parameter $\Delta / d$, Table 1 also summarizes scaling factors $s^{\prime}$ for optimal transfer time and on-resonance transfer amplitudes, as well as absolute and relative bandwidths. The scaling factor $s^{\prime}$ is defined as $\tau_{i d} / \tau_{\text {opt }}$, where $\tau_{i d}$ corresponds to the first extremum of the transfer functions under the unscaled dipolar coupling Hamiltonian $\mathrm{H} \quad d, 12$ (Eq. [1]) and $\tau_{\text {opt }}$ is the mixing time at which the first extremum of the transfer function is reached for a given multiple-pulse sequence, assuming both spins to be on 

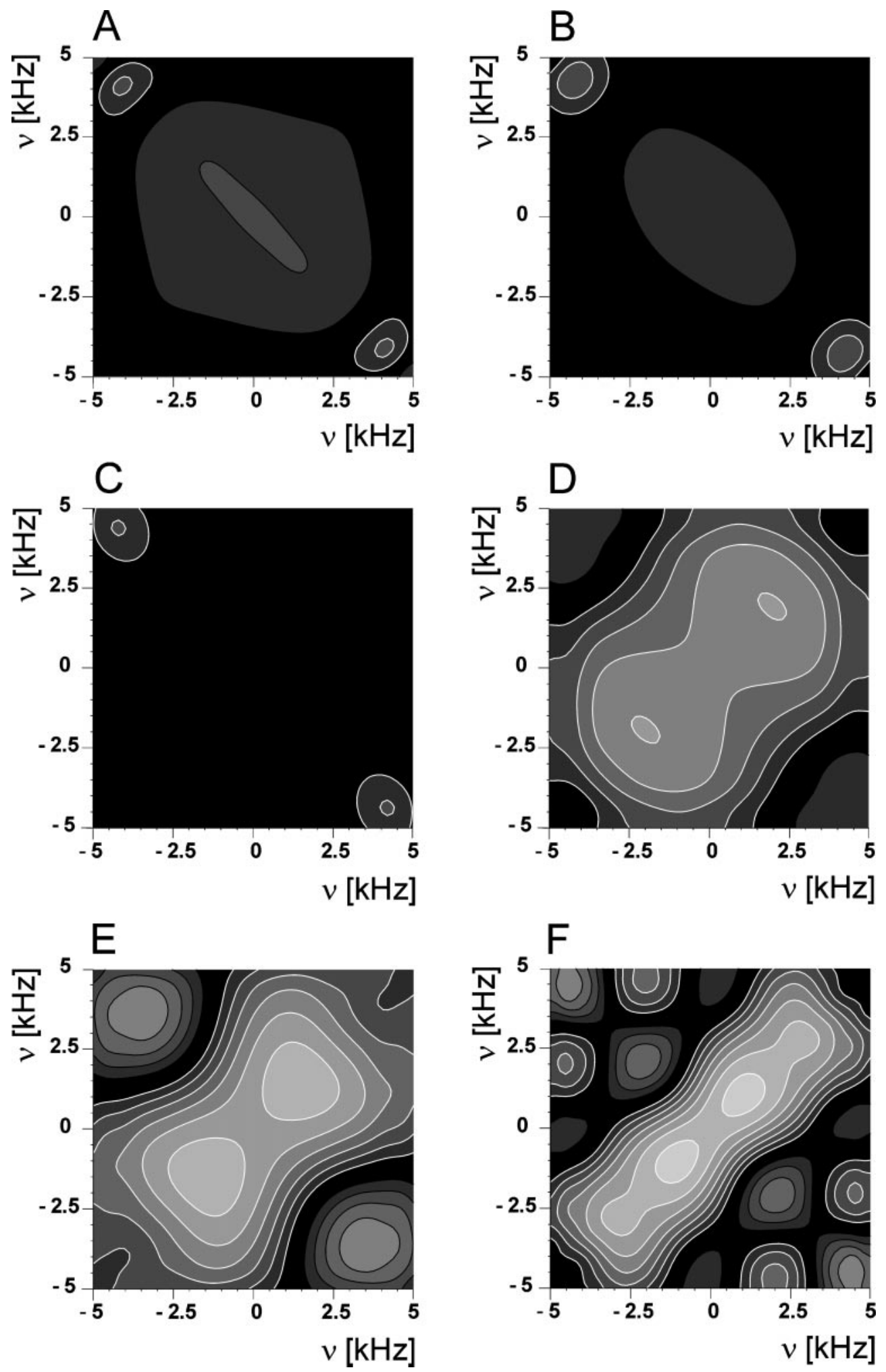

FIG. 3. Influence of the duration of the delay $\Delta$ on the offset characteristics of coherence transfer of the MOCCA-M16 sequence with a nominal rf amplitude of $12.5 \mathrm{kHz}$, corresponding to $d=40 \mu \mathrm{s}$ and a dipolar coupling constant $D_{k l}=10 \mathrm{~Hz}$. The transfer efficiency $T_{x}^{i n h}\left(0.3 / D_{k l}\right)$ is shown for the delays (A) $\Delta=$ $1 \mu \mathrm{s}$, (B) $\Delta=10 \mu \mathrm{s}$, (C) $\Delta=20 \mu \mathrm{s}$, (D) $\Delta=80 \mu \mathrm{s}$, (E) $\Delta=160 \mu \mathrm{s}$, and (F) $\Delta=320 \mu \mathrm{s}$. Black and white lines represent positive and negative contours, respectively. Contour lines are shown for $\pm 0.1, \pm 0.2, \ldots, \pm 0.9$. Areas with the same absolute value of $T_{x}^{i n h}\left(0.3 / D_{k l}\right)$ are filled by the same gray level. 


\section{TABLE 1}

MOCCA Sequences Optimized for Broadband D ipolar Transfer of $x, y$, and $z$ Magnetization

\begin{tabular}{cccccccc}
\hline & & & & \multicolumn{3}{c}{$b$} & $v_{r f}^{r m s}$ \\
$T_{\alpha}$ & \multicolumn{1}{c}{ Sequence } & $\Delta / d$ & $s^{\prime}$ & $T_{\alpha}^{o p t}$ & {$[\mathrm{kHz}]$} & {$[\mathrm{kHz}]$} & $b / v_{r f}^{r m s}$ \\
\hline$T_{x}$ & MOCCA-M16 & 3.2 & 0.75 & -0.62 & 2.5 & 6.1 & 0.41 \\
& MOCCA-M4P9 & 2.8 & 0.74 & -0.66 & 3.6 & 6.4 & 0.56 \\
& MOCCA-XY16 & 2.7 & 0.75 & -0.77 & 5.5 & 6.5 & 0.85 \\
$T_{y}$ & MOCCA-M16 & $\mathbf{2 . 2}$ & $\mathbf{0 . 7 6}$ & $-\mathbf{0 . 9 1}$ & $\mathbf{6 . 2}$ & $\mathbf{7 . 0}$ & $\mathbf{0 . 8 9}$ \\
& MOCCA-M4P9 & $\mathbf{2 . 4}$ & $\mathbf{0 . 7 5}$ & $\mathbf{- 0 . 8 6}$ & $\mathbf{7 . 0}$ & $\mathbf{6 . 8}$ & $\mathbf{1 . 0 3}$ \\
& MOCCA-XY16 & 2.7 & 0.75 & -0.77 & 5.3 & 6.5 & 0.82 \\
$T_{z}$ & MOCCA-M16 & 3.2 & 0.84 & 0.86 & 3.0 & 6.1 & 0.49 \\
& MOCCA-M4P9 & $\mathbf{2 . 4}$ & $\mathbf{0 . 7 8}$ & $\mathbf{0 . 8 9}$ & $\mathbf{4 . 0}$ & $\mathbf{6 . 8}$ & $\mathbf{0 . 5 9}$ \\
& MOCCA-XY16 & $\mathbf{2 . 2}$ & $\mathbf{0 . 7 4}$ & $\mathbf{1 . 0 0}$ & $\mathbf{5 . 5}$ & $\mathbf{7 . 0}$ & $\mathbf{0 . 7 9}$ \\
\hline
\end{tabular}

Note. An rf amplitude of $v_{r f}=12.5 \mathrm{kHz}$ and a dipolar coupling constant $D=D_{k l}=10 \mathrm{~Hz}$ were assumed in the optimization. Given are the optimized values $\Delta / d=2 v_{r f} \Delta$ for an inversion element of the form $\left[\Delta / 2-\left(180_{x}^{\circ}\right)-\right.$ $\Delta / 2$ ], expanded with XY-16 (MOCCA-XY16), MLEV-16 (MOCCA-M16), and M4P9 (MOCCA-M4P9), respectively. For example, the optimal delay for the transfer of $z$ magnetization is $\Delta=88 \mu$ s for MOCCA-XY16. In addition the parameters $s^{\prime}, T_{\alpha}^{o p t}, b, v_{r f}^{r m s}$, and $b / v_{r f}^{r m s}$ are given, which characterize the sequences with respect to transfer time, transfer amplitude, average rf power, and bandwidth (see text). The sequences with the best transfer properties for coherence and polarization transfer are indicated using boldface type.

resonance and taking into account the effect of rf inhomogeneity. This scaling factor $s^{\prime}$ based on the transfer time approaches the scaling factor $s_{k l}$ for MOCCA-XY16 which according to Eq. [9] conserves the form of the dipolar coupling term for all values of $\Delta$. For MOCCA-M16 and MOCCA-M4P9 the $s^{\prime}$ values can still be defined based on the transfer times even though Eq. [4] does not apply because the form of the dipolar coupling term is not conserved by these sequences. For example, for the transfer of $y$ magnetization $\tau_{i d}=30 \mathrm{~ms}$ (see thin dashed line in Fig. 2 B) whereas for MOCCA-M16 with $\Delta=88 \mu$ s (see Table 1) $\tau_{\text {opt }}=40 \mathrm{~ms}$, resulting in $s^{\prime}=0.76$ and $T_{y}^{i n h}\left(\tau_{\text {opt }}\right)=$ -0.91 . These values can be rationalized based on the theoretical transfer functions (Eq. [7]) with $D_{k l}^{x, e f f}=10, D_{k l}^{y, \text { eff }}=5.313$, and $D_{k l}^{z, e f f}=-15.313$ according to Eq. [5]. The absolute bandwidth $b$ is defined as the offset range of $v_{1}$ and $v_{2}$ in which the transfer amplitude $T_{\alpha}^{i n h}\left(\tau_{o p t}\right)$ is larger than 0.5 . The parameter $v_{r f}^{r m s}$ indicates the root mean square of the rf amplitude which is proportional to the square root of the average rf power of the sequences. Finally, the relative bandwidths $b / \nu_{r f}^{r m s}$ are summarized in Table 1.

For polarization transfer $\left(T_{z}\right)$ the sequence MOCCA-XY16 with $\Delta / d=2.2$ gives the best results with respect to maximum transfer amplitude $T_{z}^{o p t}$ and bandwidth $b$ (see Fig. 4A) followed by MOCCA-M4P9 with $\Delta / d=2.4$ (see Fig. 4A'). For coherence transfer, $T_{y}$ is preferable to $T_{x}$ and the sequences MOCCA-M16 with $\Delta / d=2.2$ (see Fig. 4B) and MOCCAM4P9 with $\Delta / d=2.4$ (see Fig. $4 \mathrm{~B}^{\prime}$ ) are best suited. Figure 2 also shows the detailed time dependence of the on-resonance transfer functions $T_{z}^{i n h}(\tau)$ for the optimized MOCCA-XY16 and MOCCA-M4P9 sequences and $T_{y}^{i n h}(\tau)$ for MOCCA-M16 and MOCCA-M4P9. For the optimized MOCCA sequences the transfer of magnetization (bold curves in Fig. 2) is more than $50 \%$ faster than that for DIPSI-2 (thin curves in Fig. 2). Also the maximum transfer amplitudes $\left|T_{\alpha}^{i n h}\left(\tau_{\text {opt }}\right)\right|$ of the MOCCA sequences are comparable to or even higher than the corresponding maximum transfer amplitudes of DIPSI-2 (see Fig. 2).

\section{EXPERIMENTAL}

The MOCCA and DIPSI-2 mixing sequences have been applied to an approximately $5 \mathrm{mM}$ sample of bovine red blood cell ubiquitin in a $\mathrm{H}_{2} \mathrm{O} / \mathrm{D}_{2} \mathrm{O} 90 / 10$ solution at $\mathrm{pH} 6.5$ and $303 \mathrm{~K}$ (commercially available from Sigma, Inc., St. Louis, MO, and used without further purification) in a 5-mm thin-wall tube $(550 \mu \mathrm{l})$. Orientation was induced with a dilute liquid crystal solution consisting of $3 \%$ CHAPSO/DLPC/CTAB $(1: 5: 0.1)$ (CHAPSO, DLPC, Sigma, Inc.; CTAB, Acros, New Jersey) $(26,27)$. All experiments were performed at $303 \mathrm{~K}$ on a Bruker DRX 600-MHz spectrometer (Bruker Analytik GmbH, Rheinstetten, Germany) equipped with TXI HCN z-gradient probes. Spectra were processed using XWINNMR 2.6 (Bruker Analytic $\mathrm{GmbH}$ ) and Felix 98 (MSI, San Diego, CA). The basic $z$-filter version of a 2D TOCSY is shown in Fig. 5. All TOCSY experiments (DIPSI-2, MOCCA-XY16, MOCCA-M4P9, MOCCAM16) were recorded with an rf amplitude $v_{r f}$ of $12.5 \mathrm{kHz}$. In order to achieve the maximum transfer between the amide protons which are not scalar coupled to each other, we irradiated the mixing sequences at the $\mathrm{NH}$ resonances and jumped the proton frequency back to the water resonance before detection. These frequency jumps were also performed with DIPSI-2 for better comparison. A typical dipolar mixing experiment for the transfer of $z$ magnetization is shown in Fig. 6. For different mixing sequences buildup curves as a function of the mixing time were recorded for a comparison of experimental and theoretical transfer functions (Fig. 7). The following mixing times were used: $30,60,90,120$, and $150 \mathrm{~ms}$ for $z$ transfer using MOCCA-XY16; $30,60,90$, and $120 \mathrm{~ms}$ for $z$ transfer using DIPSI-2; and 24.12, 48.24 , and $72.36 \mathrm{~ms}$ for $y$ transfer using MOCCA-M4P9. For all other mixing sequences also spectra were recorded but the ones listed performed best in the experiments, as expected from simulations. For the transfer of $z$ magnetization the maximum transfer amplitude of MOCCA-XY16 is between 14\% (B) and 61\% (A) larger than that of DIPSI-2. This difference increases dramatically for longer mixing times. At a mixing time of $120 \mathrm{~ms}$ the peaks in the MOCCA-XY16 experiment are on average a factor of 4 larger than those in the DIPSI- 2 experiment. The fastest $y$ transfer is found for MOCCA-M4P9.

\section{DISCUSSION}

New dipolar mixing sequences have been optimized and applied to a protein. If relatively small bandwidths are sufficient 

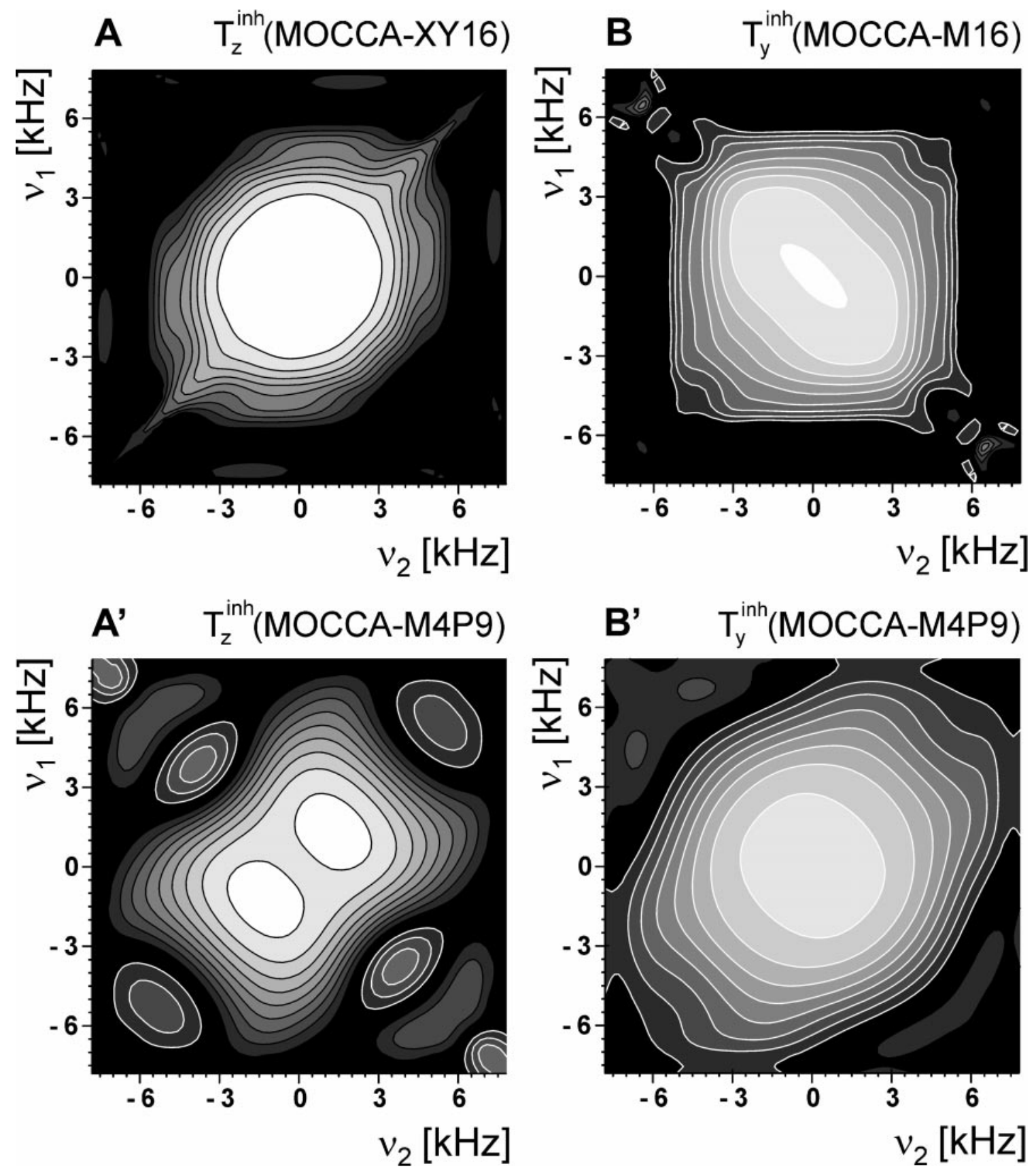

FIG . 4. Offset dependence of the transfer efficiency $T_{z}^{i n h}(0.68 / D)$ for the sequence MOCCA-XY16 (A), $T_{z}^{i n h}(0.64 / D)$ for the sequence MOCCA-M4P9 (A'), and $T_{y}^{i n h}(0.4 / D)$ for the sequences MOCCA-M16 (B) and MOCCA-M4P9 (B') with the optimized values $\Delta / d$ given in Table 1 (contour lines as in Fig. 3). The chosen mixing times $\tau_{\text {opt }}$ correspond to the first extremum of the functions $T_{\alpha}^{i n h}(\tau)$ for the respective MOCCA sequence; see Fig. 2 and Table1.

for a given application, scaling factors $s_{k l}$ of about 1 can be reached using MOCCA sequences with $\Delta \gg d$. For broadband applications, slightly reduced scaling factors have to be taken into account. For example, the MOCCA-XY16 sequence which was optimized for broadband dipolar mixing transfer (Table 1) has a maximum scaling factor $s_{k l}=0.75$ for coherence transfer and $s_{k l}=0.74$ for polarization transfer, which are about
$50 \%$ larger than the scaling factor $\left|s_{k l}\right|=0.5$ of DIPSI-2 (or WALTZ-16). These increased scaling factors of the MOCCA sequences directly translate into faster transfer dynamics (see Fig. 2 and Fig. 7). In principle, faster transfer reduces relaxation losses. However, the effective relaxation rates also depend on the trajectories of magnetization and coherences during a given multiple-pulse sequence. Based on a simple (invariant) 


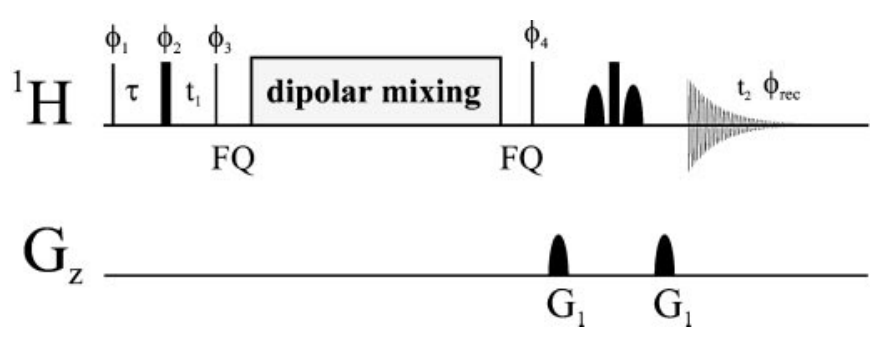

FIG. 5. The schematic pulse sequence used to test various dipolar mixing sequences for the transfer of $z$ magnetization. Narrow and wide bars represent $90^{\circ}$ and $180^{\circ}$ pulses. The default phase for pulses is $x$. Phase cycling: $\phi_{1}=x$, $-x ; \phi_{2}=y,-y ; \phi_{3}=4(x), 4(-x) ; \phi_{4}=2(x), 2(-x) ; \phi_{r e c}=x, 2(-x), x,-x$, $2(x),-x$. Quadrature detection is obtained by altering $\phi_{1}$ in the States-TPPI manner (28). During a typical repetition delay of $1.5 \mathrm{~s}$ a low-power presaturation was applied to reduce radiation damping. The delay $z=3 \mu$ s and the following $180^{\circ}$ pulse refocus chemical shift evolution during the first $t_{1}$ increment. Carrier positions: ${ }^{1} \mathrm{H}=4.65 \mathrm{ppm}$ (7.6 ppm during the dipolar mixing sequence). FQ indicates frequency jumps between the water resonance and the amide region (see text). Except for the dipolar mixing (TOCSY) sequence, proton pulses were applied using a $36.75-\mathrm{kHz}$ rf field ( $\pi$ pulse $13.6 \mu \mathrm{s}$ ). For water suppression a watergate sequence was used (29). Gradients (sine-bell shaped): $G_{1}=(1 \mathrm{~ms}$, $30 \mathrm{G} / \mathrm{cm}$ ). For the $2 \mathrm{D}$ version of the experiment 16 scans per $t_{2}$ (768 complex points, spectral width $7788 \mathrm{~Hz}$ ) experiment were recorded with 2048 complex points in $t_{2}$ (spectral width $7788 \mathrm{~Hz}$ ), giving rise to a total measurement time of about $6 \mathrm{~h}$ for each $2 \mathrm{D}$ version of the experiment.

trajectory model that ignores ZQ contributions (30, 9), the following conclusions can be drawn for characteristic cases of interest: In MOCCA-type sequences with $\Delta \gg d$ the transfer of $z$ magnetization is mainly subject to $T_{1}$ processes, whereas the transfer of $x$ or $y$ magnetization is dominated by the much faster $T_{2}$ relaxation. In DIPSI-type experiments (as well as for MOCCA sequences with $\Delta \ll d$ ) the transfer of $y$ and $z$ magnetization is subject to an average of $T_{1}$ and $T_{2}$ processes whereas the transfer of $x$ magnetization is dominated by $T_{2}$ relaxation. This qualitatively explains the observed decay rates of the ex-

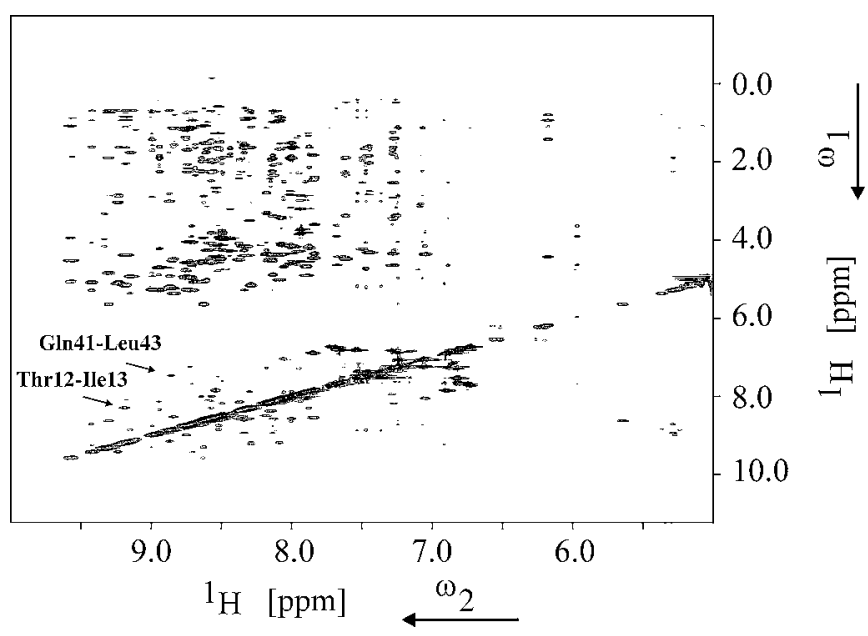

FIG . 6. ${ }^{1} \mathrm{H}^{\mathrm{N}}$ region of the MOCCA-XY16 spectrum recorded on ubiquitin. In the dipolar mixing period $z$ magnetization was transferred. The mixing period $\tau$ was $60 \mathrm{~ms}$ (experimental parameters, see text and Fig. 5).
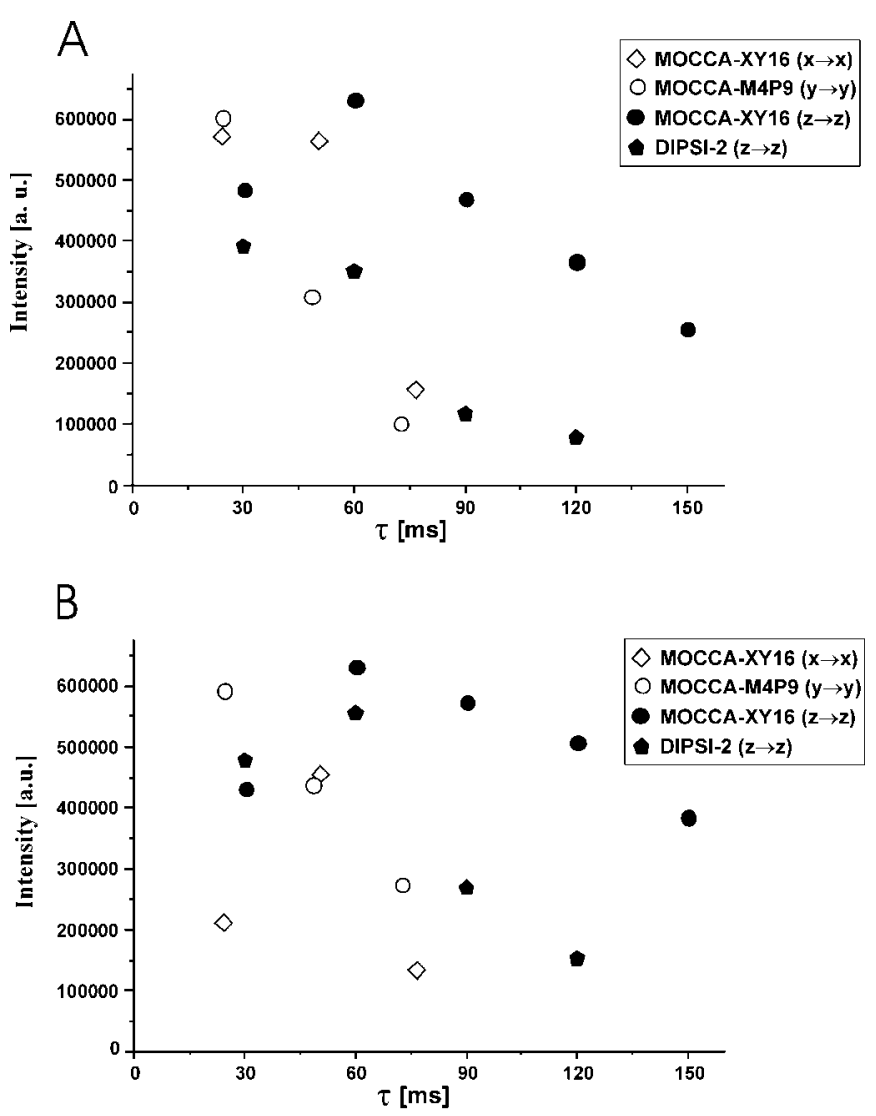

FIG. 7. Experimental dipolar transfer functions for the mixing sequences DIPSI-2 ( $z$ transfer, pentagons), MOCCA-XY16 ( $z$ transfer, black circles; $x$ transfer, diamonds) and MOCCA-M4P9 ( $y$ transfer, white circles) for the protein ubiquitin dissolved in a $\mathrm{H}_{2} \mathrm{O} / \mathrm{D}_{2} \mathrm{O} 90 / 10$ solution at $\mathrm{pH}$ 6.5. Individual crosspeak intensities (arbitrary units) are shown as a function of the mixing time $\tau$ for (A) the cross peaks between $\mathrm{H}^{\mathrm{N}}$ (Ile 13) and $\mathrm{H}^{\mathrm{N}}$ (Thr 12) with a distance of $4.46 \AA$ and (B) the cross peaks between $H^{\mathrm{N}}$ (Leu 43) and $\mathrm{H}^{\mathrm{N}}(\mathrm{Gln} 41)$ with a distance of $6.94 \AA$. In order to facilitate the comparison, the negative crosspeak intensities of the DIPSI-2, the MOCCA-M4P9, and the MOCCA-XY16 $(x \rightarrow x)$ experiments were multiplied by -1 .

perimental transfer functions in Fig. 7. In addition, the apparent decay rates are in part a result of the fact that the spin systems consist of more than two dipolar-coupled spins in which the transfer dynamics of coherence and polarization transfer can be significantly different (12). In practice, the optimized MOCCA sequences are preferable to DIPSI-type sequences due to their improved scaling properties and (in particular for the transfer of $z$ magnetization) because of their favorable effective relaxation rates, as expected based on the presented theoretical and experimental results. For the latter reason the new mixing sequences will be important building blocks in many experiments based on homonuclear dipolar transfer. For example, in $J_{\mathrm{HH}}$-NOESY experiments (31) the NOESY transfer step can be replaced by a dipolar mixing step. Relative to the applied rf power, the bandwidth of the MOCCA sequences is comparable to the bandwidth of DIPSI-2. 


\section{APPE NDIX}

A short derivation for the average Hamiltonian $\mathrm{H}_{\tilde{d}, k l}$ (see Eq. [5]) is given below based on the toggling frame approach (19). For the pulse sequence given in Fig. 1, the toggling frame Hamiltonian $\mathrm{H}_{d, k l}(t)$ is given by

$$
\mathrm{H}_{d, k l}=2 \pi D_{k l}\left\{\tilde{I}_{k x} \tilde{I}_{l x}+\tilde{I}_{k y} \tilde{I}_{l y}-2 \tilde{I}_{k z} \tilde{I}_{l z}\right\}
$$

for $0 \leq t<\Delta / 2, \Delta / 2+d \leq t<3 \Delta / 2+d$, and $3 \Delta / 2+$ $2 d \leq t<2(\Delta+d)$, and by

$$
\begin{aligned}
H \tilde{d, k l}= & 2 \pi D_{k l}\left\{\tilde{I}_{k x} \tilde{I}_{l x}+\left(\cos ^{2} \alpha-2 \sin ^{2} \alpha\right) \tilde{I}_{k y} \tilde{I}_{l y}+\left(\sin ^{2} \alpha\right.\right. \\
& \left.-2 \cos ^{2} \alpha\right) \tilde{I}_{k z} \tilde{I}_{l z}-3 \sin \alpha \cos \alpha\left(\tilde{I}_{k y} \tilde{I}_{l z}+\tilde{I}_{l y} \tilde{I}_{k z}\right)
\end{aligned}
$$

for $\Delta / 2 \leq t<\Delta / 2+d$ and $3 \Delta / 2+d \leq t<3 \Delta / 2+2 d$ with $\alpha=2 \pi v_{\mathrm{rf}}(t-\Delta / 2)$ and $\alpha=2 \pi v_{\mathrm{rf}}(t-3 \Delta / 2-d)$, respectively. The resulting average Hamiltonian, $\mathrm{H}_{d, k l}$, is given by

$$
\begin{aligned}
\tilde{\tilde{d}}_{d, k l}= & \frac{2 \pi D_{k l}}{2(d+\Delta)}\left\{2(d+\Delta) I_{k x} I_{l x}+\left[2 \Delta+\frac{d}{\pi}\right.\right. \\
& \left.\times \int_{0}^{2 \pi}\left(\cos ^{2} \alpha-2 \sin ^{2} \alpha\right) d \alpha\right] I_{k y} I_{l y} \\
& +\left[-4 \Delta+\frac{d}{\pi} \int_{0}^{2 \pi}\left(\sin ^{2} \alpha-2 \cos ^{2} \alpha\right) d \alpha\right] I_{k z} I_{l z} \\
& \left.-\frac{d}{\pi} \int_{0}^{2 \pi}(3 \sin \alpha \cos \alpha) d \alpha\left(I_{k y} I_{l z}+I_{l y} I_{k z}\right)\right\},
\end{aligned}
$$

which is identical to Eq. [5] because $\int_{0}^{2 \pi}\left(\sin ^{2} \alpha-2 \cos ^{2} \alpha\right) d \alpha=$ $\int_{0}^{2 \pi}\left(\cos ^{2} \alpha-2 \sin ^{2} \alpha\right) d \alpha=-\pi$ and $\int_{0}^{2 \pi}(3 \sin \alpha \cos \alpha) d \alpha=0$.

\section{ACKNOWLEDGMENTS}

S.J.G. thanks Fonds der Chemischen Industrie and the DFG for support (G1 203/1-6). F.K. acknowledges a stipend from the Fonds der Chemischen Industrie. C.G. thanks also the Fonds der Chemischen Industrie, the DFG, and the MPG for support for this work. W.P. is supported by a Kekule stipend of the Fonds der Chemischen Industrie. All measurements were done at the Large Scale Facility for Biomolecular NMR at the University of Frankfurt.

\section{REFERENCES}

1. J. R. Tolman, J. M. Flanagan, M. A. Kennedy, and J. H. Prestegard, Nuclear magnetic dipole interactions in field-oriented proteins: Information for structure determination in solution, Proc. Natl. Acad. Sci. USA 92, 92799283 (1995).

2. N. Tjandra and A. Bax, Direct measurement of distances and angles in biomolecules by NMR in a dilute liquid crystalline medium, Science $\mathbf{2 7 8}$, 1111-1114 (1997).
3. A. Bax and N. Tjandra, High resolution NMR of human ubiquitin in an aqueous liquid crystalline medium, J. Biomol. NMR 10, 289-292 (1997).

4. J. H. Prestegard, New techniques in structural NMR-Anisotropic interactions, Nat. Struct. Biol., NMR Suppl., 517-522 (1998).

5. M. R. Hansen, M. Rance, and A. Pardi, Observation of long-range ${ }^{1} \mathrm{H}-{ }^{1} \mathrm{H}$ distances in solution by dipolar coupling interactions, J. Am. Chem. Soc. 120, 11210-11211 (1998).

6. M. R. Hansen, P. Hanson, and A. Pardi, Filamentous bacteriophage for aligning RNA, DNA, and proteins for measurement of nuclear magnetic resonance dipolar coupling interactions, Methods Enzymol. 317, 220-240 (2000).

7. L. Braunschweiler and R. R. Ernst, Coherence transfer by isotropic mixing: Application to proton correlation spectroscopy, J. Magn. Reson. 53, 521-528 (1983).

8. D. G. Davis and A. Bax, Assignment of complex ${ }^{1} \mathrm{H}$ NMR spectra via two-dimensional homonuclear Hartmann-Hahn spectroscopy, J. Am. Chem. Soc. 107, 2820-2821 (1985).

9. S. J. Glaser and J. J. Quant, Homonuclear and heteronuclear HartmannHahn transfer in isotropic liquids, in "Advances in Magnetic and Optical Resonance” (W. S. Warren, Ed.), Vol. 19, pp. 59-252, Academic Press, San Diego (1996).

10. F. Kramer, B. Luy, and S. J. Glaser, Offset dependence of homonuclear Hartmann-Hahn transfer based on residual dipolar couplings in solution state NMR, Appl. Magn. Reson. 17, 173-187 (1999).

11. O. Schedletzky and S. J. Glaser, Analytical coherence transfer functions for the general AMX system under isotropic mixing, J. Magn. Reson. A 123, 174-180 (1996).

12. B. Luy and S. J. Glaser, Analytical polarization and coherence transfer functions for three dipolar coupled spins 1/2, J. Magn. Reson. 142, 280-287 (2000).

13. B. H. Meier, Polarization transfer and spin diffusion in solid-state NMR, in "Advances in Magnetic and Optical Resonance" (W. S. Warren, Ed.), Vol. 18, pp. 1-116, Academic Press, San Diego (1994).

14. G. M. Clore, A. M. Gronenborn, and A. Bax, A robust method for determining the magnitude of the fully asymmetric alignment tensor of oriented macromolecules in the absence of structural information, J. Magn. Reson. 133, 216-221 (1998).

15. M. H. Levitt, Symmetrical composite pulse sequences for NMR population inversion. I. Compensation of radio frequency field inhomogeneity, J. Magn. Reson. 48, 234-264 (1982).

16. A. J. Shaka, J. Keeler, T. Frenkiel, and R. Freeman, An improved sequence for broadband decoupling: WALTZ-16, J. Magn. Reson. 52, 335-338 (1983).

17. A. J. Shaka, C. J. Lee, and A. Pines, Iterative shemes for bilinear operators: Application to spin decoupling, J. Magn. Reson. 77, 274-293 (1988).

18. H. Y. Carr and E. M. Purcell, Effects of diffusion on free precession in nuclear magnetic resonance experiments, Phys. Rev. 94, 630-638 (1954).

19. U. Haeberlen, "Advances in Magnetic Resonance, Suppl. 1" (J. S. Waugh, Ed.), p. 68, Academic Press, New York (1976).

20. R. R. Ernst, G. Bodenhausen, and A. Wokaun, "Principles of Nuclear Magnetic Resonance in One and Two Dimensions," p. 75, Oxford Univ. Press, New York (1987).

21. T. Gullion, D. B. Baker, and M. S. Conradi, New, compensated Carr-Purcell sequences, J. Magn. Reson. 89, 479-484 (1990).

22. M. J. Lizak, T. Gullion, and M. S. Conradi, Measurement of like-spin dipole couplings, J. Magn. Reson. 91, 254-260 (1991).

23. R. Tycko and A. Pines, Fixed point theory of iterative excitation schemes in NMR, J. Chem. Phys. 83, 2775-2802 (1985). 
24. H. M. Cho, R. Tycko, and A. Pines, Iterative maps for bistable excitation of two-level systems, Phys. Rev. Lett. 56, 1905-1908 (1985).

25. S. J. Glaser and G. P. Drobny, Assessment and optimization of pulse sequences for homonuclear isotropic mixing, in "Advances in Magnetic and Optical Resonance" (W. S. Warren, Ed.), Vol. 14, pp. 35-58, Academic Press, San Diego (1990).

26. J. A. Losonczi and J. H. Prestegard, Improved dilute bicelle solutions for high-resolution NMR of biological macromolecules, J. Biomol. NMR 12, 447-451 (1998).

27. H. Wang, M. Eberstadt, T. Olejniczak, R. P. Meadows, and S. W. Fesik, A liquid crystalline medium for measuring residual dipolar couplings over a wide range of temperatures, J. Biomol. NMR 12, 443-446 (1998).
28. D. Marion, M. Ikura, R. Tschudin, and A. Bax, Rapid recording of 2D NMR spectra without phase cycling. Application to the study of hydrogen exchange in proteins, J. Magn. Reson. 85, 393-399 (1989).

29. M. Piotto, V. Saudek, and V. Sklenár, Gradient-tailored excitation for single-quantum NMR spectroscopy of aqueous solutions, J. Biomol. NMR 2, 661-665 (1992).

30. C. Griesinger and R. R. Ernst, Cross relaxation in time-dependent nuclear spin systems: Invariant trajectory approach, Chem. Phys. Lett. 152, 239-247 (1988).

31. W. Peti and C. Griesinger, Measurement of magnitude and sign of H,H dipolar couplings in proteins, J. Am. Chem. Soc. 122, 3975-3976 (2000). 